\title{
BMJ Open Cohort profile: molecular signature in pregnancy (MSP): longitudinal high- frequency sampling to characterise cross-omic trajectories in pregnancy in a resource-constrained setting
}

\author{
Tobias Brummaier (D) , ${ }^{1,2,3,4}$ Basirudeen Syed Ahamed Kabeer, ${ }^{5}$ \\ Pornpimon Wilaisrisak, ${ }^{1}$ Mupawjay Pimanpanarak, ${ }^{1}$ Aye Kyi Win, ${ }^{1}$ \\ Sasithon Pukrittayakamee, ${ }^{6,7}$ Alexandra K Marr, ${ }^{5}$ Tomoshige Kino, ${ }^{5}$ \\ Souhaila Al Khodor, ${ }^{5}$ Annalisa Terranegra, ${ }^{5}$ Verena I Carrara, ${ }^{1,2}$ \\ Francois Nosten (D) , ,2 Jürg Utzinger, ${ }^{3,4}$ Damien Chaussabel, ${ }^{5}$ Daniel H Paris, ${ }^{3,4}$ \\ Rose McGready ${ }^{1,2}$
}

To cite: Brummaier T, Syed Ahamed Kabeer B, Wilaisrisak $P$, et al. Cohort profile: molecular signature in pregnancy (MSP): longitudinal high-frequency sampling to characterise cross-omic trajectories in pregnancy in a resourceconstrained setting. BMJ Open 2020;10:e041631. doi:10.1136/ bmjopen-2020-041631

- Prepublication history for this paper is available online. To view these files, please visit the journal online (http://dx.doi. org/10.1136/bmjopen-2020041631).

Received 13 June 2020

Revised 31 August 2020

Accepted 03 September 2020

Check for updates

(C) Author(s) (or their employer(s)) 2020. Re-use permitted under CC BY-NC. No commercial re-use. See rights and permissions. Published by BMJ.

For numbered affiliations see end of article.

Correspondence to

Dr Tobias Brummaier;

tobias.brummaier@gmx.at

\section{ABSTRACT}

Purpose A successful pregnancy relies on the interplay of various biological systems. Deviations from the norm within a system or intersystemic interactions may result in pregnancy-associated complications and adverse pregnancy outcomes. Systems biology approaches provide an avenue of unbiased, in-depth phenotyping in health and disease. The molecular signature in pregnancy (MSP) cohort was established to characterise longitudinal, crossomic trajectories in pregnant women from a resource constrained setting. Downstream analysis will focus on characterising physiological perturbations in uneventful pregnancies, pregnancy-associated complications and adverse outcomes.

Participants First trimester pregnant women of Karen or Burman ethnicity were followed prospectively throughout pregnancy, at delivery and until 3 months post partum. Serial high-frequency sampling to assess whole blood transcriptomics and microbiome composition of the gut, vagina and oral cavity, in conjunction with assessment of gene expression and microbial colonisation of gestational tissue, was done for all cohort participants.

Findings to date 381 women with live born singletons averaged 16 (IQR 15-18) antenatal visits (13094 biological samples were collected). At 5\% (19/381) the preterm birth rate was low. Other adverse events such as maternal febrile illness $7.1 \%$ (27/381), gestational diabetes $13.1 \%$ (50/381), maternal anaemia $16.3 \%(62 / 381)$, maternal underweight $19.2 \%(73 / 381)$ and a neonate born small for gestational age $20.2 \%$ (77/381) were more often observed than preterm birth.

Future plans Results from the MSP cohort will enable in-depth characterisation of cross-omic molecular trajectories in pregnancies from a population in a resource-constrained setting. Moreover, pregnancyassociated complications and unfavourable pregnancy outcomes will be investigated at the same granular level, with a particular focus on population relevant needs such as effect of tropical infections on pregnancy. More detailed
Strengths and limitations of this study

The major strength is the prospective nature of the study and frequent follow-up, coupled with highfrequency sampling and thus, availability of detailed clinical information and a considerable number of biological samples.

- High-throughput analysis, in combination with clinical data, will enable investigation of a number of pregnancy-related physiological and pathological changes.

- Frequently populations from low-resource settings are disproportionally burdened by adverse birth outcomes that may be based on exposure to different communicable diseases; hence, including them in high-end clinical research, addresses a significant research gap and may result in improvements of limited relevance to high-income countries.

- Low numbers for some phenotypes (eg, preterm birth) may prove to be detrimental for the validity of observed differences; although the power will depend on the magnitude of observed differences in molecular signatures.

- In low-resource settings, complete biological sample sets are often difficult to obtain, which may downsize the richness of the data.

knowledge on multiomic perturbations will ideally result in the development of diagnostic tools and ultimately lead to targeted interventions that may disproportionally benefit pregnant women from this resource-limited population. Trial registration number NCT02797327.

\section{INTRODUCTION}

A successful pregnancy relies on welltimed adaptations and the interplay of multiple maternal biological systems. These 
interactions and temporal changes affect various organ systems, such as the cardiovascular, respiratory, endocrine system or metabolic systems, and, more recently, pivotal immunological adaptions and changes in the human microbiome became evident. ${ }^{1-5}$ Physiological adaptions of the immune system during pregnancy play a central role in implantation and placentation, promotion of fetal growth and initiation of labour and delivery. ${ }^{2}$ Deviations from the norm of this fine-tuned immune clock may lead to dysregulation in biological networks and cause various pregnancy-associated complications with their immediate consequences for the mother and fetus. ${ }^{6}$

With a growing body of evidence of the human microbiome's significance in health and disease, investigating its role in reproductive medicine has opened up an avenue to a deeper understanding of adverse pregnancy outcomes. ${ }^{7}$ The placenta represents the fetomaternal link. Its crucial role during pregnancy is underlined by the fact that failure in its development and function is associated with a variety of pregnancy-associated complications (eg, pre-eclampsia, intrauterine growth restriction and preterm birth, PTB). ${ }^{8}$

In recent years, investigation of these biological systems has greatly improved our understanding of their role in healthy pregnancies and in pregnancies resulting in unfavourable outcomes. However, there is a need for longitudinal, multiomics profiling studies that may further contribute to the understanding of physiological adaptions, the interconnections of various biological systems and their significance in pregnancy-associated complications. ${ }^{3}$

Research, in particular high-end research, is often biased towards high-income countries. ${ }^{9}$ This imbalance is aggravated by the fact that, due to population based differences, results are often not generalisable, ${ }^{10}$ populations living in resource-constrained settings are more affected by the consequences of pregnancy-associated complications (eg, inadequate access to safe iatrogenic birth or lack of advanced neonatal care) and have different epidemiological patterns of communicable diseases. Consequently, pregnant women in low-income settings would benefit disproportionately from early identification of pregnancy-associated complications and targeted interventions.

Thus, the aim of establishing this prospective molecular signature in pregnancy (MSP) cohort was to characterise cross-omic trajectories in pregnant women from a resource-limited setting and describe pregnancyassociated complications (eg, PTB, gestational diabetes mellitus (GDM), anaemia, underweight and born too small for gestational age (SGA)). The term 'molecular signature' was chosen, as it refers to molecular markers that can be used for in-depth description of a particular phenotype. ${ }^{11}$

Perturbations related to the immune response will be investigated by measuring the abundance of RNAs in circulating nucleated cells (ie, leucocytes) via capillary blood sampling at multiple time points. ${ }^{12}$ Microbiome profiling of the intestinal and vaginal niche in pregnancy, at delivery and post partum will be complemented by assessment of the oral microbiome. Lastly, placental tissue will be investigated for the presence of bacterial colonisation as well as for the relationship between the genome structure and changes in global patterns of gene expression. The generated molecular data will then be analysed in the context of clinical and patient related data. As described in the study protocol, ${ }^{13}$ an immediate focus was the investigation of PTB.

The MSP cohort profile represents the link between development of the study protocol and the results. Accordingly, cohort characteristics, including the recruitment and follow-up process, demographics, pregnancy outcomes, biological samples available for analysis and preliminary results, are presented.

\section{COHORT DESCRIPTION}

\section{Setting and participants}

Women with an unremarkable medical and obstetric history attending the antenatal care (ANC) facilities of Shoklo Malaria Research Unit (SMRU), a field station of the Faculty of Tropical Medicine at Mahidol University (Bangkok, Thailand) that is part of the Mahidol-Oxford Research Unit, were invited to participate. SMRU operates free-of-charge walk-in services for marginalised migrant populations in clinics at the frontier of Thailand with Myanmar (figure 1). This mobile population mostly resides in small, rural villages. The major source of income in this community comes from work in the agricultural sector or as daily labourers, while about one in four women stay at home to look after the household. ${ }^{14}$ As most of these daily employments provide only minimum wages coupled with the often undocumented legal status, the living conditions of most pregnant women in this community are very basic. ${ }^{15}$ The official minimal wages in Myanmar are around MMK4800 (US\$3) per day, and in Thailand around THB300 (US\$9) per day.

Between September 2016 and July 2018, trained counsellors from the community provided general information about the trial to all pregnant women who registered at SMRU ANC. Detailed information, including visual aids, was used to illustrate the purpose, procedures and timing of follow-up. Potential risks and benefits of the study were explained to women meeting the following inclusion criteria: (1) pregnant woman willing and able to give written informed consent; (2) Karen or Burman ethnicity; (3) age 18-49 years; (4) healthy, with viable singleton first trimester $(8+0$ to $<14+0$ weeks) pregnancy; (5) planning to deliver at the SMRU birth unit and (6) able and willing to comply with study requirements. If these criteria were met and appropriate consent was provided, women were followed from the first trimester, throughout pregnancy, at delivery and until 3 months post partum.

It was estimated that approximately 400 women could be enrolled to build the cohort from this low-resource environment when considering constraints of clinical capacity 


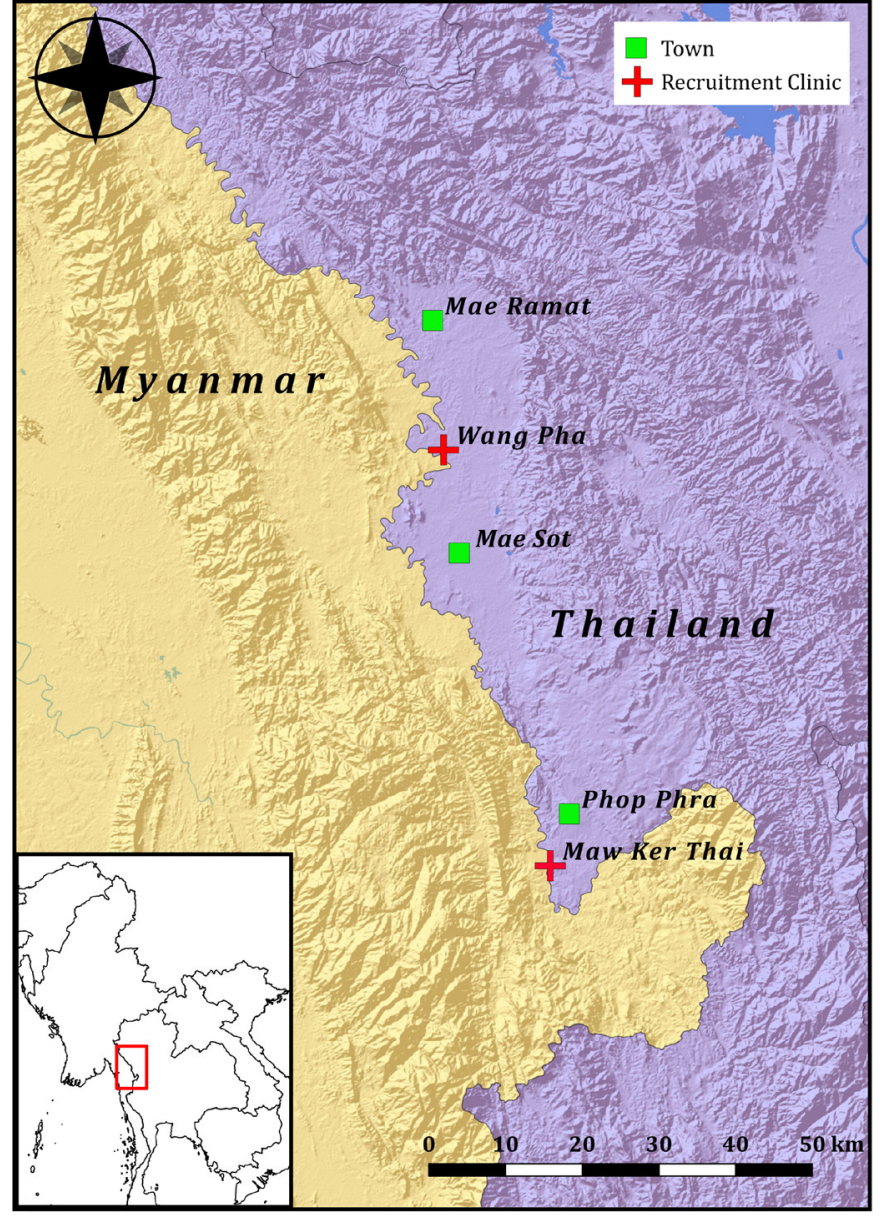

Figure 1 Setting and location of recruitment clinics.

and budget availability. With this sample size, events such as PTB (estimated at $8 \%$, that is, 30 cases expected) would be feasible but also provide scope to engage with other pregnancy-associated complications, tropical infections (eg, small liver fluke infections estimated at $5 \%$, that is, 20 cases expected) and febrile illness (estimated at $10 \%$, that is, an expectation of 40 cases) with a sufficient number of uneventful pregnancies remaining to act as controls.

Figure 2 provides an overview of the number of participants recruited and followed. During the active recruitment period, 4292 pregnant women registered to ANC. Seventy per cent $(2544 / 3609)$ were ineligible due to late presentation to ANC. There were two local conditions that limited recruitment. The first issue pertained to access; pregnant women following SMRU ANC come from villages scattered along the Thailand-Myanmar border (figure 1) with transportation constraints affecting their ability to attend, further aggravated in the monsoon season as roads become impassable. The second issue related to staffing. As only one ultrasound machine was available at each site, there was an upper limit to the number of women who could have study related fetal growth scans done on a single day. Challenges in access and staffing issues led to the non-consideration of 178 and 475 women, respectively.
Number of women enrolled in SMRU ANC

programme during the study recruitment period $(n=4,292)$

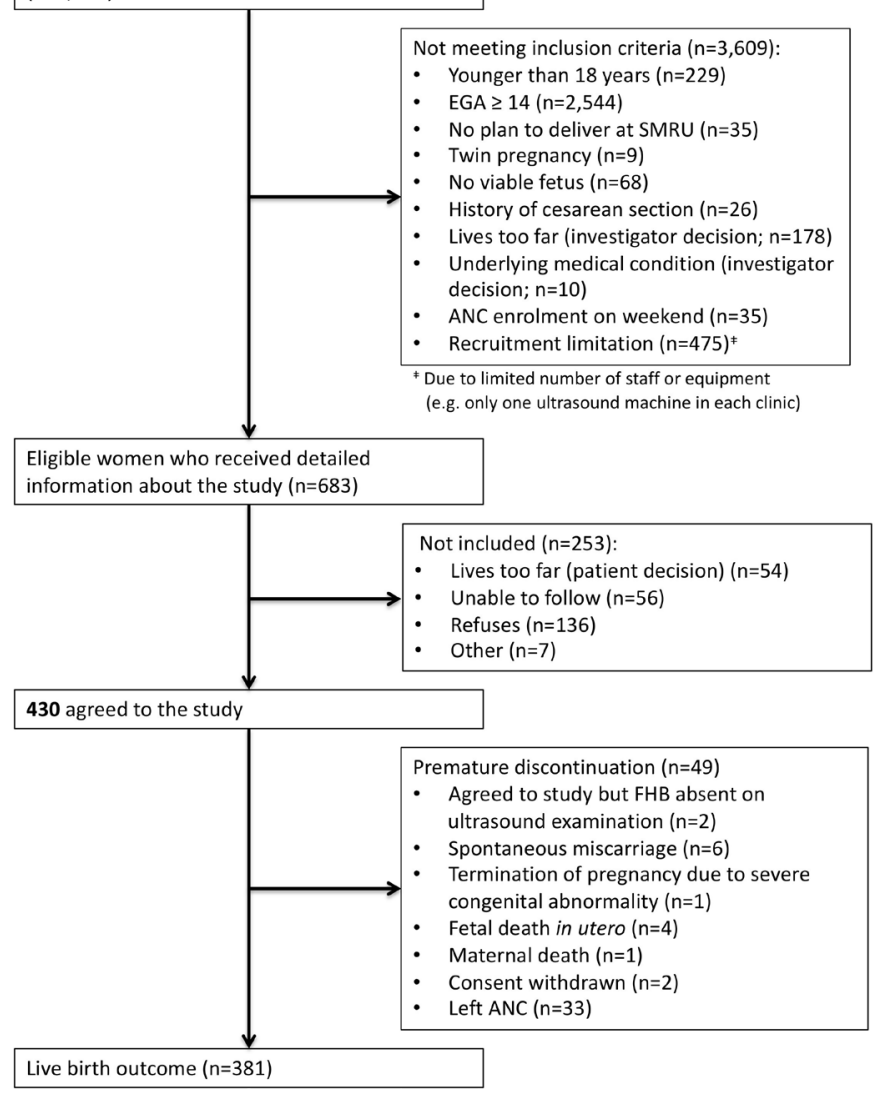

Figure 2 Screening, enrolment and outcome flow chart. ANC, antenatal care; EGA, estimated gestational age; FHB, fetal heartbeat; SMRU, Shoklo Malaria Research Unit.

Overall, 683 women received full information about the purpose and procedures of the study. Of these, 430 agreed to participate (response rate: 63\%) and consented by providing a signature or thumbprint if the event of an illiterate participant. Thirty participants were recruited in the cohort to replace women who were lost before delivery, while the study was still open to recruitment. Two participants were excluded as the fetal heartbeat (FHB) was absent after they had already agreed to the study (figure 2).

Routine ANC procedures at SMRU are described in the study protocol. ${ }^{13}$ Demographic characteristics of 381 enrolled women with a live birth outcome are summarised (table 1). To demonstrate the scope of the cohort, selected subgroups are summarised alongside the characteristics for all 381 women.

\section{Participant follow-up}

Women were followed every 2 weeks during pregnancy, at delivery and monthly in the first 3 months after delivery. Trial participants were encouraged to report to the study clinic if they experienced an adverse event, with special consideration for febrile episodes to maximise information on the nature of the experienced morbidity and its effect on the pregnancy. ${ }^{13}$ Overall, 6443 study visits 
Table 1 Basic characteristics of women with a live birth outcome $(n=381)$ and subgroups of selected pregnancy-associated complications in the molecular signature in pregnancy cohort

\begin{tabular}{|c|c|c|c|c|c|c|}
\hline & $\begin{array}{l}\text { Overall } \\
(n=381)\end{array}$ & $\begin{array}{l}\text { Preterm } \\
(\mathrm{n}=19)\end{array}$ & $\begin{array}{l}\text { GDM } \\
(n=50)\end{array}$ & $\begin{array}{l}\text { Anaemia } \\
(n=62)\end{array}$ & $\begin{array}{l}\text { Underweight } \\
(n=73)\end{array}$ & $\begin{array}{l}\text { SGA } \\
(n=77)\end{array}$ \\
\hline Inclusion EGA (days); median (range) & $82(56-97)$ & $83(60-94)$ & $82.5(56-97)$ & $81(60-94)$ & $81(57-95)$ & $81(62-97)$ \\
\hline \multicolumn{7}{|l|}{ Maternal age groups (years) } \\
\hline $18-24$ & $185(48.6)$ & $14(73.7)$ & $25(50.0)$ & $28(45.2)$ & $43(58.9)$ & 38 (49.4) \\
\hline $25-29$ & 95 (24.9) & $2(10.5)$ & $12(24.0)$ & $14(22.6)$ & $18(24.7)$ & $18(23.4)$ \\
\hline $30-34$ & $64(16.8)$ & $3(15.8)$ & $8(16.0)$ & $12(19.4)$ & $10(13.7)$ & $13(16.9)$ \\
\hline $35-39$ & $25(6.6)$ & 0 & $4(8.0)$ & $5(8.1)$ & $2(2.7)$ & $6(7.8)$ \\
\hline $40-49$ & $12(3.1)$ & 0 & $1(2.0)$ & $3(4.8)$ & 0 & $2(2.6)$ \\
\hline \multicolumn{7}{|l|}{ Residence } \\
\hline Myanmar & $263(69.0)$ & $10(52.6)$ & $33(66.0)$ & $42(67.7)$ & $43(58.9)$ & $52(67.5)$ \\
\hline Thailand & $118(31.0)$ & $9(47.4)$ & $17(34.0)$ & $20(32.3)$ & $18(24.7)$ & $25(32.5)$ \\
\hline \multicolumn{7}{|l|}{ Gravidity } \\
\hline Primigravida & $103(27.0)$ & $8(42.1)$ & $12(24.0)$ & $17(27.4)$ & $32(43.8)$ & $26(33.8)$ \\
\hline 2 & $111(29.1)$ & $8(42.1)$ & $16(32.0)$ & $21(33.9)$ & $21(28.8)$ & $18(23.4)$ \\
\hline 3 & $79(20.7)$ & $2(10.5)$ & $12(24.0)$ & $14(22.6)$ & $9(12.3)$ & $13(16.9)$ \\
\hline 4 & $47(12.3)$ & $1(5.3)$ & $7(14.0)$ & $4(6.5)$ & $8(11.0)$ & $11(14.3)$ \\
\hline$\geq 5$ & $41(10.8)$ & 0 & $3(6.0)$ & $6(9.7)$ & $3(4.1)$ & $9(11.7)$ \\
\hline \multicolumn{7}{|l|}{ Parity } \\
\hline 0 (nullipara) & $127(33.3)$ & $9(47.4)$ & $16(32.0)$ & $23(37.1)$ & $37(50.7)$ & $32(41.6)$ \\
\hline 1 & $117(30.7)$ & $8(42.1)$ & $15(30.0)$ & $18(29.0)$ & $18(24.7)$ & $16(20.8)$ \\
\hline 2 & $70(18.4)$ & $1(5.3)$ & $11(22.0)$ & $14(22.6)$ & $11(15.1)$ & $13(16.9)$ \\
\hline 3 & $39(10.2)$ & $1(5.3)$ & $7(14.0)$ & $2(3.2)$ & $4(5.5)$ & $11(14.3)$ \\
\hline$\geq 4$ & $28(7.3)$ & 0 & $1(2.0)$ & $5(8.1)$ & $3(4.1)$ & $5(6.5)$ \\
\hline Literate & $244(64.0)$ & $13(68.4)$ & $29(58.0)$ & $43(69.4)$ & $53(72.6)$ & $51(66.2)$ \\
\hline Smoking during in pregnancy & $29(7.6)$ & 0 & $6(12.0)$ & $5(8.1)$ & $4(5.5)$ & $10(13.0)$ \\
\hline \multicolumn{7}{|l|}{ Obstetric history ${ }^{*}$} \\
\hline Miscarriage & $94(33.8)$ & $2(18.2)$ & $11(28.9)$ & 15 (33.3) & $13(31.7)$ & $19(37.3)$ \\
\hline Stillbirth & $4(1.4)$ & 0 & 0 & $1(2.2)$ & 0 & 0 \\
\hline $\mathrm{PIH}$ & $2(0.7)$ & 0 & 0 & $1(2.2)$ & 0 & 0 \\
\hline Preterm rupture of membranes & $8(2.9)$ & $2(18.2)$ & 0 & $3(6.7)$ & $1(2.4)$ & $1(2.0)$ \\
\hline Preterm labour & $23(8.3)$ & $6(54.5)$ & 0 & $9(20.0)$ & $3(7.3)$ & $3(5.9)$ \\
\hline Vacuum delivery & $3(1.1)$ & 0 & 0 & $1(2.2)$ & $1(2.4)$ & 0 \\
\hline Postpartum haemorrhage & $9(3.2)$ & 0 & 0 & $3(6.7)$ & $2(4.9)$ & $2(3.9)$ \\
\hline Retained placenta & $1(0.4)$ & 0 & 0 & 0 & 0 & 0 \\
\hline Previous neonatal death & $9(3.2)$ & $3(27.3)$ & 0 & $3(6.7)$ & 0 & $1(2.0)$ \\
\hline History of GDM & $2(0.7)$ & 0 & $2(5.3)$ & 0 & 0 & 0 \\
\hline Macrosomia & $2(0.7)$ & 0 & $1(2.6)$ & 0 & 0 & 0 \\
\hline
\end{tabular}

Basic characteristics of pregnant women who completed per protocol follow-up with a live birth outcome, compared between various pregnancy-associated complications and adverse pregnancy outcomes. One study participant may be represented in multiple subgroups.

Data presented as proportion $\mathrm{n}(\%)$ or median (range).

${ }^{*}$ Proportions calculated based on multigravida $(n=278)$ as denominator.

EGA, estimated gestational age; GDM, gestational diabetes; PIH, pregnancy induced hypertension; SGA, born too small for gestational age.

during pregnancy were recorded, of which 6140 were from women with a live birth outcome. The median number of ANC visits for women with a live birth was 16
(IQR 15-18). In the uncommon event of cohort participants attending other health facilities, diagnosis and treatment was recorded at the next visit. All participants 
were encouraged to deliver at SMRU under the care of a skilled birth attendant. Women from the cohort achieved a low rate of home birth $6.6 \%(25 / 381)$ in contrast to the estimated 7 in 10 births that take place at home in rural Myanmar. ${ }^{16}$

As an estimator for adherence to study protocol, the number of expected and actual samples provided by women with a known pregnancy outcome were calculated. No significant difference was noted when comparing the proportion of expected and actual samples between capillary blood, stool and vaginal swab samples $(\mathrm{p}=0.070$, $\mathrm{p}=0.767$ and $\mathrm{p}=0.919$, respectively). Missed follow-ups were the main reason for less than expected samples being provided. Additional samples were provided from participants who experienced a febrile illness, and on a few occasions, samples were erroneously taken as they were not required by the study protocol.

Early termination from the study occurred in $11.0 \%$ $(47 / 428)$ of pregnancies, most of whom left ANC before birth $70.2 \%$ (33/47) due to migration. Adverse pregnancy outcomes were recorded in $25.5 \%(12 / 47)$ cases and two $(4.3 \%)$ participants withdrew consent, as they no longer wanted to provide vaginal swab samples (figure 2).

\section{Data collection}

Data covering three major aspects were collected, namely (1) clinical data; (2) genome-wide transcript abundance and (3) analysis of microbial composition in various anatomical sites. Table 2 summarises all study relevant procedures.

Routine ANC procedures included physical and obstetric examination, recording of concomitant medications and 6-weekly ultrasound scans to monitor fetal growth. Clinical data were collected, so that trajectories of maternal and fetal physical change would be available by the study endpoint. Two-weekly gestational weight, fetal growth and haematocrit were done as malnutrition and anaemia (and formerly malaria) are prevalent pregnancyassociated morbidities. ${ }^{17-19}$

Ultrasound scans to monitor fetal growth were performed 6-weekly. Throughout the study period, 2850 scans were done; 2693 of these were scans in women with a known, viable birth outcome (157 scans accounted for other pregnancy outcomes).

Additional details describing the study conduct and routine ANC procedures are available elsewhere. ${ }^{13}$ Briefly, capillary blood, faecal and vaginal swab samples

\begin{tabular}{|c|c|c|c|c|c|c|}
\hline & Screening & Baseline & Follow-up & $\begin{array}{l}\text { Unwell } \\
\text { episode }\end{array}$ & Birth & Post partum \\
\hline Viable singletons pregnancy & $\mathbf{x}$ & & & & & \\
\hline Obstetric ultrasound ${ }^{*}$ & $\mathbf{x}$ & $\mathbf{x}$ & $\mathbf{x}$ & & & \\
\hline Eligibility assessment & $\mathbf{x}$ & & & & & \\
\hline Written informed consent & & $\mathbf{x}$ & & & & \\
\hline Demographics & & $\mathrm{x}$ & & & & \\
\hline Medical and obstetric history & & $\mathbf{x}$ & & & & \\
\hline Concomitant medications & & $\mathbf{x}$ & $\mathbf{x}$ & $\mathbf{x}$ & $\mathbf{x}$ & $\mathbf{x}$ \\
\hline Physical examination & & $\mathrm{x}$ & $\mathbf{x}$ & $\mathbf{x}$ & $\mathbf{x}$ & $\mathbf{x}$ \\
\hline $\begin{array}{l}\text { Universal pregnancy screening, for example, thick and } \\
\text { thin blood film for malaria diagnosis, CBC and OGTT }\end{array}$ & & $\mathbf{x}$ & & & & $(\mathbf{X}) \dagger$ \\
\hline Sample maternal $100 \mu \mathrm{L}$ capillary blood & & $\mathbf{x}$ & $x \S$ & $\mathbf{x} \boldsymbol{\eta}$ & $\mathbf{X}^{\star \star}$ & $\mathbf{x}+\dagger$ \\
\hline $\begin{array}{l}\text { Sample vaginal swab, stool specimen and } 24 \text { hours } \\
\text { food recall }\end{array}$ & & $\mathbf{x} \neq \ddagger$ & $\mathbf{X} \neq \ddagger$ & $\mathbf{x}$ & $\mathbf{x}$ & $\mathbf{x} \S \S$ \\
\hline Acceptability survey & & $\mathbf{x}$ & & & & $\mathbf{x}$ \\
\hline Sample saliva & & & xशी & & $x$ & \\
\hline $\begin{array}{l}\text { Sample placenta, cord blood and maternal venous } \\
\text { blood }\end{array}$ & & & & & $\mathbf{x}$ & \\
\hline
\end{tabular}

${ }^{*}$ Fetal growth scans on a 6-weekly basis.

†OGTT at 24-26 weeks of gestation; repeated at 12 weeks post partum if positive during pregnancy.

$\ddagger 50 \mu \mathrm{L}$ for whole blood transcriptome analysis and $50 \mu \mathrm{L}$ for haematocrit.

§2-weekly; if the woman attended all expected 15 visits total blood is $1.5 \mathrm{~mL}$.

IIf the woman attended for an unwell visit, an additional $100 \mu \mathrm{L}$ of blood were drawn.

**If delivery at SMRU clinic, then an additional $100 \mu \mathrm{L}$ of blood were drawn.

††At 1, 2 and 3 months postpartum, including maternal haematocrit.

¥¥In each trimester of pregnancy: 8-14, 20-22 and 34-35 weeks.

$\S \S$ Vaginal swab samples at 4-6 weeks and at 3 months.

१ๆAt 24-26 weeks of gestation.

CBC, complete blood count; OGTT, oral glucose tolerance test; SMRU, Shoklo Malaria Research Unit. 
were taken during pregnancy, at delivery and in the postpartum period. To assess the influence of nutrition on the faecal microbiome, each faecal sample was coupled with a 24-hour food recall (table 2). In the event of a febrile episode, an additional set of these samples were taken together with a standardised fever screening battery. Oral microbiome was assessed in the second trimester and at delivery. Placental tissue samples were taken at delivery along with umbilical cord blood serum and maternal serum.

As SMRU birthing units do not have capacity for caesarean sections, participants were referred to the closest public hospitals when indicated, and hence, delivery samples were not available, in common with births at home or in other health facilities. Even though gestational tissue samples were not available for these participants, outcome measures and neonatal anthropometry were mostly available.

Lastly, in an effort to understand the acceptance of the high-frequency sampling in combination with the dense follow-up schedule and the perception of invasiveness of sampling procedures, an acceptability survey at study enrolment and completion was conducted.

\section{Biological samples}

Standard operating procedures (SOPs) focused on detailed description of the sample collection procedures were drafted before study commencement and are available on request. To accommodate the high number of samples, a unique barcoded sample sticker was placed on each tube, containing the participants study code that was assigned at enrolment, as well as date and time when samples were collected. All samples were transferred from the study sites to SMRU's central laboratory daily and stored according to SOPs. Freezerworks (Dataworks Development, Mountlake Terrace, Washington, USA), a biorepository software was used for sample management and tracking.

Including women lost to follow-up and pregnancies that resulted in a miscarriage or stillbirth, 13536 biological samples were taken between study enrolment and 3-month post partum. Considering multiple aliquots for vaginal swab samples (four aliquots at each sampling time point), saliva samples $(n=4)$ and placenta samples $(n=12)$, the total number of samples available for testing is 25816 .

Table 3 Overview of available biological samples for women with a live birth outcome $(n=381)$ and selected subgroups of the molecular signature in pregnancy cohort

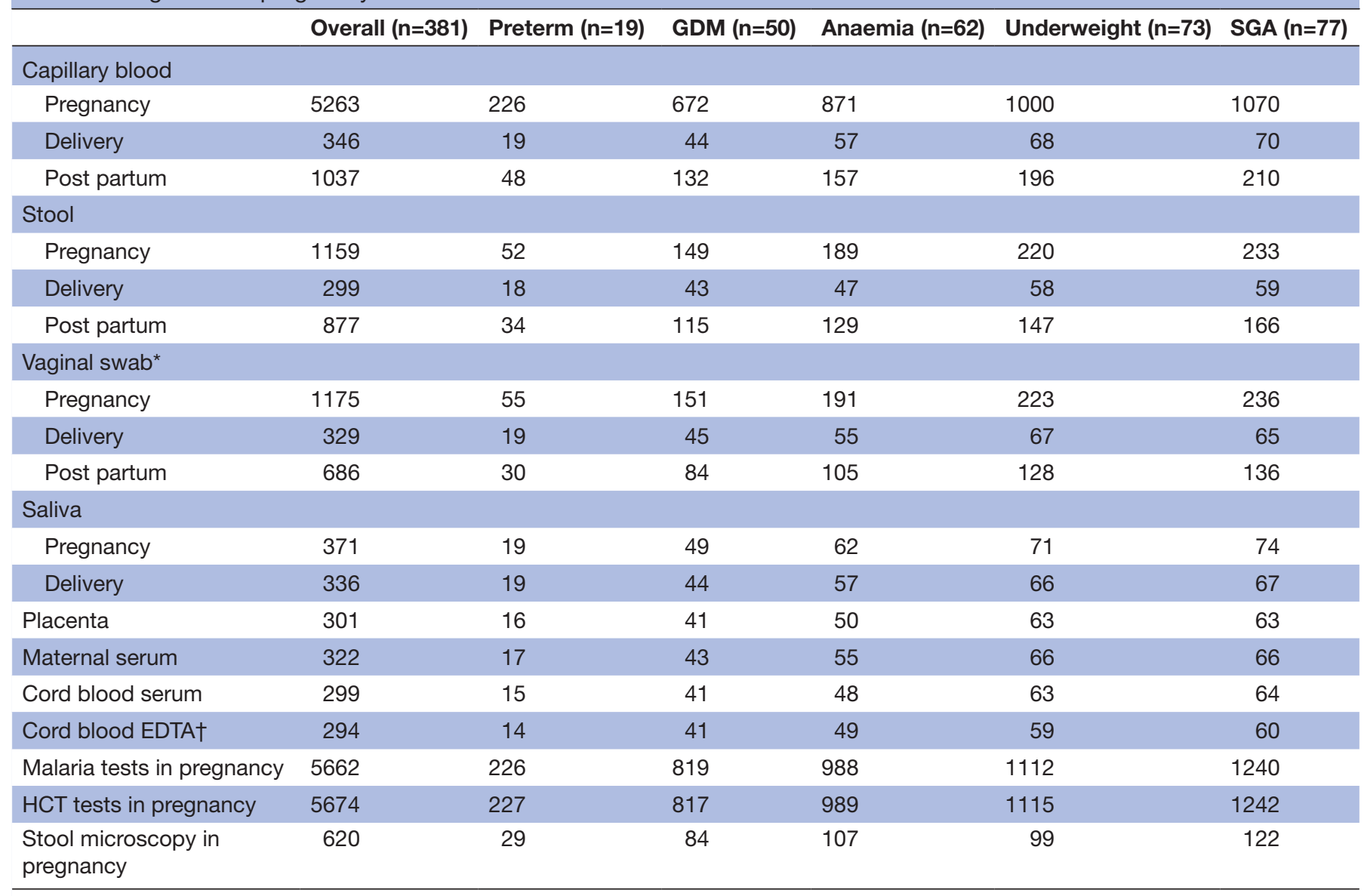

*Number refers to sampling time points; four swabs were taken at each time point.

†Less samples as amended to the protocol at a later stage.

EDTA, ethylenediaminetetraacetic acid; GDM, gestational diabetes mellitus; HCT, haematocrit; SGA, born too small for gestational age. 


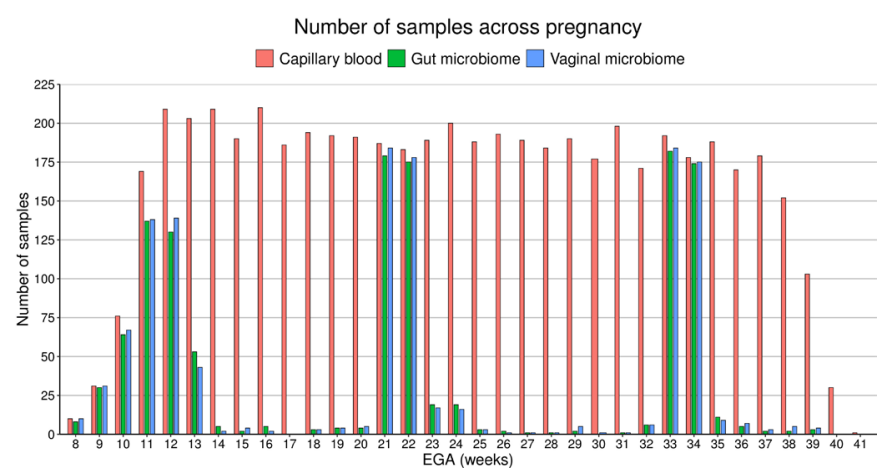

Figure 3 Number of samples over the course of pregnancy by week of gestation. EGA, estimated gestational age.

Table 3 provides an overview of biological samples available for analysis and figure 3 depicts the distribution of capillary blood, faecal and vaginal swab samples in relation to the estimated gestation of pregnancy.

All biological samples will be analysed and interpreted in the context of clinical data. To investigate physiological trajectories and deviations from these in the event of pregnancyassociated complications, a nested case-control approach will be applied, and potential confounding factors will be adjusted for. Results will be published in original research articles alongside more detailed background information pertaining to the respective content knowledge, precise methods of sampling procedures and analytical steps of respective laboratory-based methods to enable the scientific community to draw their conclusions on appropriateness. The MSP cohort profile and the previously published study protocol $^{13}$ will serve as a cross-reference for all results.

The molecular signatures pertaining to biological samples will be analysed by collaborators at Sidra Medicine (Doha, Qatar). To assure appropriate sample handling, shipment is delegated to World Courier (AmerisourceBergen; Chesterbrook, Pennsylvania, USA), a leading, global specialty and logistics provider. All samples are kept on dry ice and shipped in freezer boxes with thick styrofoam walls that are equipped with temperature monitors. At the time of writing, 16916 biological samples have been successfully transferred.

\section{Participant involvement}

Study participants were not directly involved in the study design, the elaboration of research questions and outcome measures or the recruitment and conduct of the study. However, the protocol was reviewed and approved by a local community advisory board whose members are representatives of the local community and act as a bridge between researchers and the local population. ${ }^{20}$ Their role was to advise on ethical and operational aspects of the study and they will provide a channel to inform the community of the results of this study.

\section{FINDINGS TO DATE}

Demographic data, findings during pregnancy and relevant outcome data for the overall cohort population with a live birth are presented in tables 1 and 4, respectively. According to study objectives, data of commonly observed pregnancy-associated complications (GDM, anaemia and underweight) and adverse pregnancy outcomes (PTB and SGA) are presented alongside the overall cohort.

At a rate of $5.0 \%(19 / 381)$, PTB (defined as delivery before 37 weeks of gestation), was considerably less than the worldwide estimate of approximately $10.6 \%$ (uncertainty interval $9.0 \%-12.0 \%$ ) of live-born infants. ${ }^{21}$ When considering all pregnancies followed at SMRU during the study period, the observed proportion of PTB was roughly $6.8 \%$, a number still below the worldwide average. The estimated gestational age (EGA) at delivery of PTB cases ranged from $30^{+2}$ to $36^{+6}$ (weeks ${ }^{\text {+days }}$ ) with the majority of cases $(89.5 \%)$ being moderate/late PTB (i.e. $\geq 32$ and $<37$ weeks of gestation). Of the 19 PTB cases, one was iatrogenic with induction for severe preeclampsia at an EGA of $36^{+3}$, while the remainder were spontaneous, without any discernible causative factor. Prioritising healthy women at the outset and frequent visits with healthcare providers might have contributed to earlier identification of health issues and timely intervention. Population-based factors that might be associated with the low PTB rate are the low rate of sexually transmitted diseases, and preliminary data suggest that prevalence of Gardnerella vaginalis, a facultative anaerobic coccobacillus that is associated with PTB, is low in this population. This low PTB rate is unique in itself and will be thoroughly assessed by a holistic investigation of available demographic, clinical and cross-omic data.

There were six spontaneous miscarriages (median EGA $16^{+3}\left(\right.$ IQR $\left.16^{+1}-17^{+2}\right)$ ) and one pregnancy was terminated due to a congenital abnormality (severe hydrops fetalis) at an EGA of $20^{+1}$. There were four stillbirths; all were identified antepartum as intrauterine fetal deaths before completion of 37 weeks of gestation. One was unexplained with an absent FHB at $28^{+1}$ weeks, one was associated with pre-eclampsia (absent $\mathrm{FHB}$ at $31^{+1}$ ), one had a tight cord around the neck at birth (absent FHB at $35^{+3}$ ), and in one case the mother ingested organophosphate with suicidal intent (absent FHB at $36^{+3}$ ). One unexpected neonatal death was recorded in a term neonate born to a mother with an unremarkable pregnancy and delivery.

Seven of $381(1.8 \%)$ women experienced Plasmodium vivax malaria during pregnancy and one woman was diagnosed with $P$. vivax malaria at delivery. Soiltransmitted helminth infections are common in this population and were detected in $21.3 \%(81 / 381)$ of pregnant women with a live birth outcome. Hookworm was detected in $15.0 \%(57 / 381)$ cases, Trichuris trichiura in $6.3 \%(24 / 381)$ and Ascaris lumbricoides in $4.2 \%(16 / 381)$. Fourteen women had more than one soil-transmitted helminth infection concurrently. Foodborne trematode infections (eg, small liver flukes) were detected in $6.8 \%(26 / 381)$ of the participants. The immunological fingerprint of helminthic infections and their effect on pregnancy is poorly understood. Hence, effects of helminth infections on the immune system or the intestinal microbiome in pregnancy will be studied with data from this cohort. 


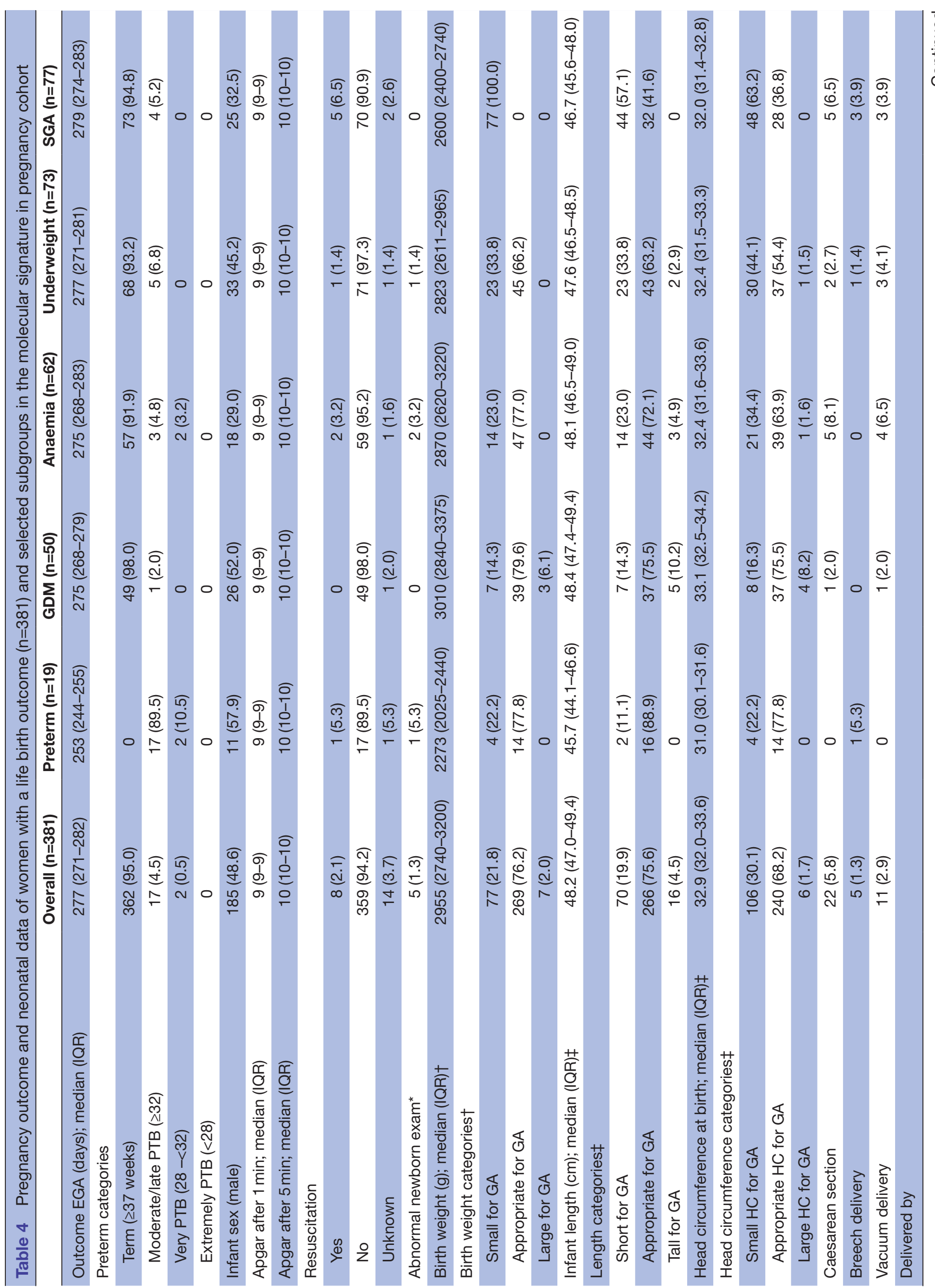

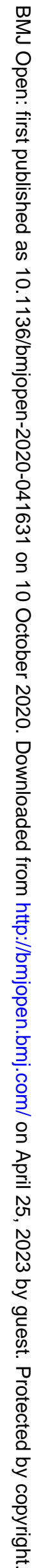




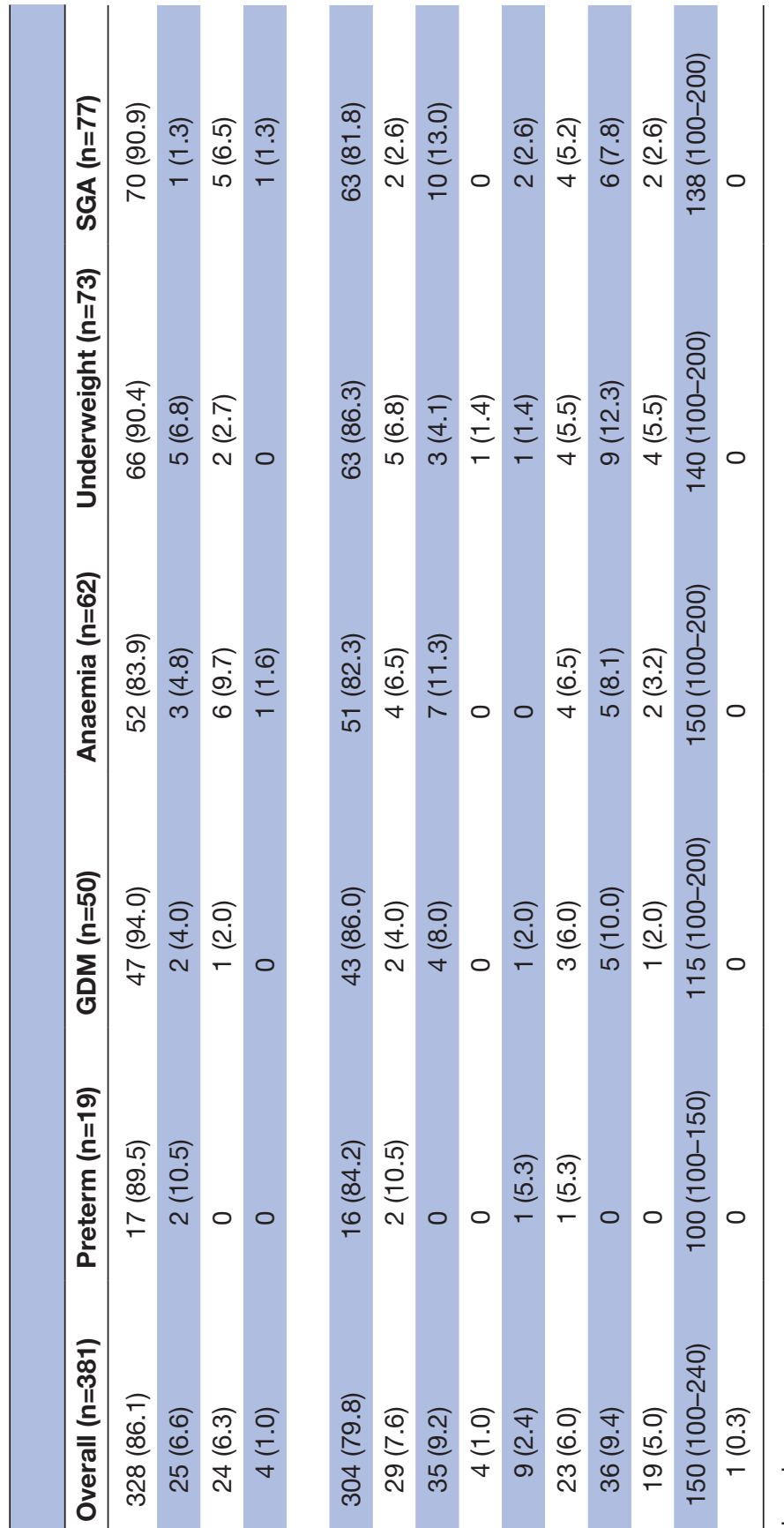

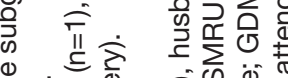
요워

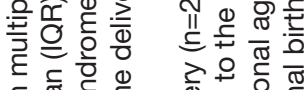

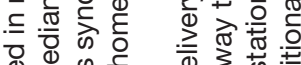

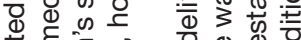

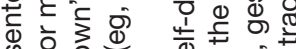

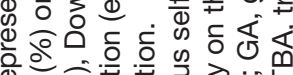
잉 ه

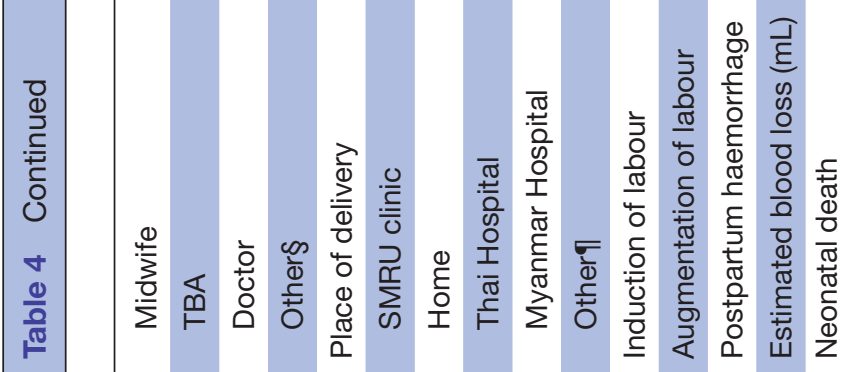

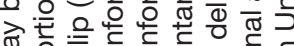

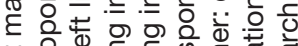

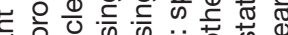
ब 表元

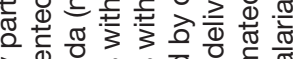

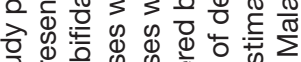
至产

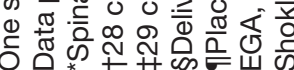


Generally, communicable diseases are often associated with adverse pregnancy outcomes. Fever, which is often the cardinal symptom of an infectious disease, was recorded in $7.1 \%(27 / 381)$ of pregnant women. Whether longitudinal, high-frequency sampling has potential for early detection, or detection prior to the onset of symptoms, of communicable diseases in pregnancy, will be determined. Availability of data pertaining to communicable disease will also allow appropriate adjustment for investigation of other objectives (eg, PTB).

Overall, only four cases $(1.0 \%)$ of preeclampsia and one case $(0.3 \%)$ of eclampsia were recorded which is also at the lower end of the epidemiological range (0.2-9.2). ${ }^{22}$ A common pregnancy-associated complication was GDM, which affected $13.1 \%(50 / 381)$ pregnancies with a live birth. GDM is the most common metabolic disorder in pregnancy and is of increasing concern in high-income settings around the globe but also in this resourcelimited population. ${ }^{23}{ }^{24}$ While the proportion of pregnant women diagnosed with underweight in this population is decreasing, $19.2 \%(73 / 381)$ of pregnancies were still complicated by a diagnosis of underweight. ${ }^{19}$ Maternal anaemia defined as haematocrit of less than $30 \%$ complicated $16.3 \%(62 / 381)$ of pregnancies and SGA $(20.2 \%$ $(77 / 381))$ according to standards as published by the Intergrowth-21st consortium ${ }^{25}$ was also commonly observed and remains a solicitude in this pregnant population. As shown in figure 3, cohort enrolment in early pregnancy, coupled with the high-frequency sampling provides a plethora of cross-omic data. This will enable to identify signatures preceding the development of these complications, their impact on pregnancy outcome and to characterise how they plateau in the postpartum period.

Ultralow capillary blood samples are safe and a reliable method to monitor longitudinal gene expression trajectories. Samples acquired from this MSP cohort were used to develop a modified SOP for extraction of RNA from small volume whole blood samples preserved in Tempus

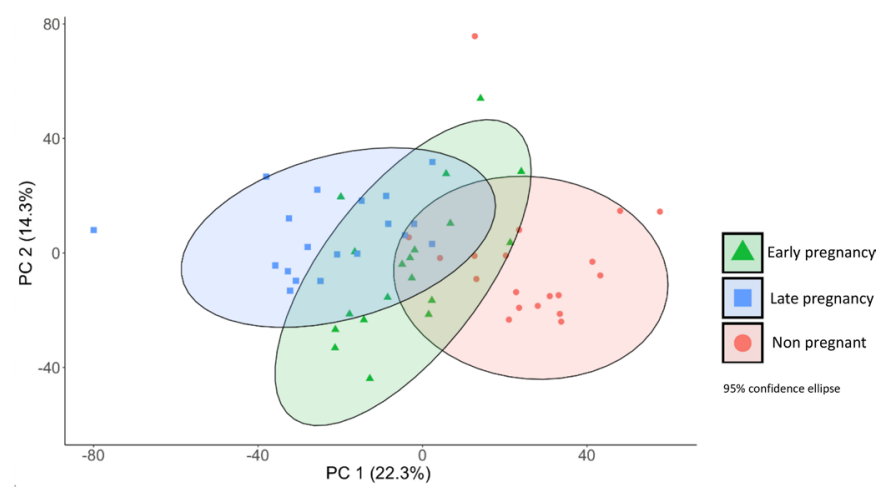

Figure 4 Principal component $(\mathrm{PC})$ analysis of whole blood gene expression data (RNAseq) of 19 uneventful, term pregnancies of the MSP cohort compared between first trimester (early pregnancy), third trimester (late pregnancy) and 3-month post partum (non-pregnant). MSP, molecular signature in pregnancy. solution ${ }^{26}$ and the performance of ultralow blood samples was benchmarked previously. ${ }^{27}$ Figure 4 shows a comparison between different stages in pregnancy (early pregnancy and late pregnancy), and the non-pregnant control (3-month post partum) by exploratory analysis of gene expression patterns (RNASeq) in 19 MSP women with uneventful, term pregnancies. The preliminary finding of clustering of different timepoints in pregnancy and post partum, respectively, follows patterns reported by Ngo et $a l$ who were able to predict EGA by analysing cell free RNA transcripts in maternal blood. ${ }^{28}$

\section{STRENGTHS AND LIMITATIONS}

The major strength of this cohort is the prospective and the frequent follow-up along the trajectory from first trimester to 3 months post partum. Coupled with the availability of a large number of well characterised biological samples, the high-throughput analysis, in combination with clinical data, we anticipate that a number of pregnancy-related physiological and pathological changes can be investigated and described in detail. Systematic approaches enable the researchers to assess the entirety of a biological system and are thought to minimise the bias introduced through selective parameters.

Populations originating from low-resource settings do not often have the same level of access to advanced research when compared with high-income settings. While some data on intestinal microbiome are available, ${ }^{29}$ data generated from the MSP cohort will characterise composition and perturbations of the vaginal microbiome for the first time in this population.

The small number of PTB was not anticipated and is lower than rates reported internationally. While a larger figure would be desirable from an analytical point of view, comparison of molecular markers and associated population-based factors to populations with a higher risk of PTB will provide novel insight for this low observed proportion. Generally, a selection and exclusion bias at participant enrolment might have contributed to the low number of PTB, as women with an unremarkable medical and obstetric history were given priority to minimise the risk of potential lost to follow-up and subsequent sample loss. Hence, inference on the power of the result cannot be made at this point, as the power will depend on the magnitude of observed differences in molecular signatures between term and preterm pregnancies.

More faecal samples were missed compared with capillary blood or vaginal swab samples. In an effort to reduce sample loss for potential future research, a nested project was conceived to assess whether collection and transfer of fresh faecal samples in the long-term storage tube at home is feasible, sample quality is affected, and acceptability of self-collection is favourable.

Larger cohorts would be preferable to address critical research questions such as PTB. However, with the high-frequency sampling and the overall cost of the study conduct and sample analysis, the researchers enrolled 
not more participants than deemed to be necessary to address the objectives of this project.

\section{COLLABORATION}

Proposals for collaborations and other ideas to enhance scientific output from data generated in this cohort are welcome. SMRU is part of the Mahidol Oxford Tropical Medicine Research Unit and follows data sharing policies, as published by the Bioethics and Engagement Department. Data from the MSP cohort will be made available through the Data Access Committee at Mahidol Oxford Tropical Medicine Research Unit. Data sharing policies are available at: http:/ /www.tropmedres.ac/datasharing. An application form can be downloaded under this link. Gene expression data will be deposited on the NCBI Gene Expression Omnibus and data from 16S rRNA sequencing will be stored as a bioproject in NCBI for public Sequence Read archives.

\author{
Author affiliations \\ ${ }^{1}$ Shoklo Malaria Research Unit, Mahidol-0xford Tropical Medicine Research Unit, \\ Faculty of Tropical Medicine, Mahidol University, Mae Sot, Tak, Thailand \\ ${ }^{2}$ Centre for Tropical Medicine and Global Health, Nuffield Department of Medicine, \\ University of Oxford, Oxford, UK \\ ${ }^{3}$ Swiss Tropical and Public Health Institute, Basel, Switzerland \\ ${ }^{4}$ University of Basel, Basel, Switzerland \\ ${ }^{5}$ Sidra Medicine, Doha, Qatar \\ ${ }^{6}$ Mahidol Oxford Tropical Medicine Research Unit, Faculty of Tropical Medicine, \\ Mahidol University, Bangkok, Thailand \\ ${ }^{7}$ Department of Clinical Tropical Medicine, Faculty of Tropical Medicine, Mahidol \\ University, Bangkok, Thailand
}

Acknowledgements We would like to express our gratitude to the cohort participants: without the contribution of the women following ANC visits at SMRU this work would not be possible. Moreover, we do acknowledge the continuous effort of staff in the recruitment centres for the excellent participant management and their pivotal role in data collection.

Collaborators The MSP cohort is a collaboration between Shoklo Malaria Research Unit, a field station of the Faculty of Tropical Medicine, Mahidol University (Bangkok, Thailand) that is part of the Mahidol-0xford Research Unit; Sidra Medicine (Doha, Qatar), which is part of Qatar Foundation for Education, Science and Community Development; and the Swiss Tropical and Public Health Institute, an associated institute of the University of Basel (Basel, Switzerland).

Contributors All authors listed contributed substantially to this manuscript in accordance with the ICMJE criteria.

Funding This work was supported by Sidra Medicine, a member of the Qatar Foundation for Education, Science and Community Development. The Shoklo Malaria Research Unit is part of the Wellcome Trust Mahidol University Oxford Tropical Medicine Research Programme supported by the Wellcome Trust of Great Britain (Major Overseas Programme).

Map disclaimer The depiction of boundaries on the map(s) in this article does not imply the expression of any opinion whatsoever on the part of BMJ (or any member of its group) concerning the legal status of any country, territory, jurisdiction or area or of its authorities. The map(s) are provided without any warranty of any kind, either express or implied.

Competing interests None declared.

Patient consent for publication Not required.

Provenance and peer review Not commissioned; externally peer reviewed.

Data availability statement Data are available on reasonable request. All data relevant to the study are included in the article. Deidentified data from the MSP cohort will be accessible through the Data Access Committee at Mahidol Oxford
Tropical Medicine Research Unit. Gene expression data and data from 16S rRNA sequencing will be deposited on public platforms.

Open access This is an open access article distributed in accordance with the Creative Commons Attribution Non Commercial (CC BY-NC 4.0) license, which permits others to distribute, remix, adapt, build upon this work non-commercially, and license their derivative works on different terms, provided the original work is properly cited, appropriate credit is given, any changes made indicated, and the use is non-commercial. See: http://creativecommons.org/licenses/by-nc/4.0/.

ORCID iDs

Tobias Brummaier http://orcid.org/0000-0002-9739-2084

Francois Nosten http://orcid.org/0000-0002-7951-0745

\section{REFERENCES}

1 Soma-Pillay P, Nelson-Piercy C, Tolppanen H, et al. Physiological changes in pregnancy. Cardiovasc J Afr 2016;27:89-94.

2 Mor G, Aldo P, Alvero AB. The unique immunological and microbial aspects of pregnancy. Nat Rev Immunol 2017;17:469-82.

3 Peterson LS, Stelzer IA, Tsai AS, et al. Multiomic immune clockworks of pregnancy. Semin Immunopathol 2017.

4 Koren O, Goodrich JK, Cullender TC, et al. Host remodeling of the gut microbiome and metabolic changes during pregnancy. Cell 2012;150:470-80.

5 Maclntyre DA, Chandiramani M, Lee YS, et al. The vaginal microbiome during pregnancy and the postpartum period in a European population. Sci Rep 2015;5:8988.

6 Aghaeepour N, Ganio EA, Mcilwain D, et al. An immune clock of human pregnancy. Sci Immunol 2017;2. doi:10.1126/sciimmunol. aan2946. [Epub ahead of print: 01 Sep 2017].

7 Ursell LK, Metcalf JL, Parfrey LW, et al. Defining the human microbiome. Nutr Rev 2012;70:S38-44.

8 Morgan TK. Role of the placenta in preterm birth: a review. Am J Perinatol 2016;33:258-66.

9 Acharya KP, Pathak S. Applied research in low-income countries: why and how? Front Res Metr Anal 2019;4.

10 Spielman RS, Bastone LA, Burdick JT, et al. Common genetic variants account for differences in gene expression among ethnic groups. Nat Genet 2007;39:226-31.

11 Nilsson R, Björkegren J, Tegnér J. On reliable discovery of molecular signatures. BMC Bioinformatics 2009;10:38.

12 Chaussabel D, Pascual V, Banchereau J. Assessing the human immune system through blood transcriptomics. BMC Biol 2010;8:84.

13 Brummaier T, Syed Ahamed Kabeer B, Lindow S, et al. A prospective cohort for the investigation of alteration in temporal transcriptional and microbiome trajectories preceding preterm birth: a study protocol. BMJ Open 2019;9:e023417.

14 Fellmeth G, Plugge EH, Carrara V, et al. Migrant perinatal depression study: a prospective cohort study of perinatal depression on the Thai-Myanmar border. BMJ Open 2018;8:e017129.

15 Pudpong N, Durier N, Julchoo S, et al. Assessment of a voluntary Non-Profit health insurance scheme for migrants along the ThaiMyanmar border: a case study of the migrant fund in Thailand. Int $J$ Environ Res Public Health 2019;16:2581.

16 Han SM, Rahman MM, Rahman MS, et al. Progress towards universal health coverage in Myanmar: a national and subnational assessment. Lancet Glob Health 2018;6:e989-97.

17 McLean E, Cogswell M, Egli I, et al. Worldwide prevalence of anaemia, who vitamin and mineral nutrition information system, 1993-2005. Public Health Nutr 2009;12:444-54.

18 Gilder ME, Simpson JA, Bancone G, et al. Evaluation of a treatment protocol for anaemia in pregnancy nested in routine antenatal care in a limited-resource setting. Glob Health Action 2019;12:1621589.

19 Hashmi AH, Solomon N, Lee SJ, et al. Nutrition in transition: historical cohort analysis summarising trends in under- and overnutrition among pregnant women in a marginalised population along the Thailand-Myanmar border from 1986 to 2016. Br J Nutr 2019;121:1413-23.

20 Cheah PY, Lwin KM, Phaiphun L, et al. Community engagement on the Thai-Burmese border: rationale, experience and lessons learnt. Int Health 2010;2:123-9.

21 Chawanpaiboon S, Vogel JP, Moller A-B, et al. Global, regional, and national estimates of levels of preterm birth in 2014: a systematic review and modelling analysis. Lancet Glob Health 2019;7:e37-46.

22 Umesawa M, Kobashi G. Epidemiology of hypertensive disorders in pregnancy: prevalence, risk factors, predictors and prognosis. Hypertens Res 2017;40:213-20. 
23 Gilder ME, Zin TW, Wai NS, et al. Gestational diabetes mellitus prevalence in Maela refugee cAMP on the Thai-Myanmar border: a clinical report. Glob Health Action 2014;7:23887.

24 Chiefari E, Arcidiacono B, Foti D, et al. Gestational diabetes mellitus: an updated overview. J Endocrinol Invest 2017;40:899-909.

25 Villar J, Cheikh Ismail L, Victora CG, et al. International standards for newborn weight, length, and head circumference by gestational age and sex: the newborn cross-sectional study of the INTERGROWTH21st project. Lancet 2014;384:857-68.
26 Tomei S, Mattei V. A protocol for extraction of total RNA from finger stick whole blood samples preserved with TempusTM solution. F1000Research 2018;7:1739.

27 Speake C, Whalen E, Gersuk VH, et al. Longitudinal monitoring of gene expression in ultra-low-volume blood samples self-collected at home. Clin Exp Immunol 2017;188:226-33.

28 Ngo TTM, Moufarrej MN, Rasmussen M-LH, et al. Noninvasive blood tests for fetal development predict gestational age and preterm delivery. Science 2018;360:1133-6.

29 Vangay P, Johnson AJ, Ward TL, et al. US immigration Westernizes the human gut microbiome. Cell 2018;175:962-72. 